\title{
Application of Network Simulation Tool in Computer Network Teaching
}

\author{
Bo Yang \\ College of Mathematics and Finance, Sichuan University of Arts and Science, Dazhou, 635000, \\ China
}

Key words: Computer, Network teaching, Network simulation tool

\begin{abstract}
The main purpose of computer network teaching is to let students more fully know and master computer network structure, working principles and basic definition etc. as well as master how to apply computer network in practical life. This paper analyzes defects and problems of current computer network, and mainly studies network simulation technology and rational application of simulation technology in computer network teaching so as to improve continually actual classroom effect of network teaching, enrich teaching mode, expand students' view and motivate students' enthusiasm for learning computer network technology.
\end{abstract}

\section{Introduction}

With the development of modernization course, industrialization and informatization drive social progress, and computer network receives more and more attention. To continuously meet social actual demand and boost talent training intensity, many colleges start to form computer network course, but most computer network courses just utilize textbook knowledge or classroom learning in teaching process. Analysis and learning of computer network just stays on the surface. Corresponding equipment and experiment may be used during learning. Because the network owns certain particularity, this will shield operating principle to certain degree. So, bottom-layer operating principle of network cannot be known more deeply. Aiming at the above problems, network simulation technology becomes an important solution.

\section{Development status of computer network teaching}

Top-down and bottom-up teaching methods should be combined organically. Such computer teaching methods can be applied in practical teaching. Teachers need to follow top-down hierarchy when introducing computer network, and utilize common network to let students fully know actual operation functions and principles of computer network.

Case teaching method. In computer network teaching process, real teaching case is Chinese education network by which teachers explain and analyze IP/TCP working process and principles for students.

Panorama teaching method. To let students know computer network system from multiple aspects, historical method should be used to gradually analyze computer network technology. The design method should be changed from details to ensure comprehensive analysis of computer network technology ${ }^{[1]}$.

\section{Basic overview of network simulation technology}

Network simulation technology mainly simulates networks according to statistic analysis and mathematical modeling, establishes corresponding networking protocol, equipment, protocol model and chain, simulates equipment transmission process, ensures optimization of network design property and guarantees verification correctness of networking protocol. By use of case simulation platform of network simulation technology, computer's fundamental, algorithm and protocol can be basically analyzed to make sure students can more fully know core network technology. Students' learning initiative can improve. Besides, the degree of network principles can be deepened. In accordance with network simulation platform, network analog nay be constructed on a large scale. 
Students may properly analyze and understand large-scale network and learn new and old algorithms or protocols by contrast and boost the significance of learning computer network. The major case of network situation application is Packet Tracer5.00. Such software was issued by Cisco Company in 2008. When this software is applied, Cisco IOS order may be applied. Meanwhile, computer parameters may be rationally configured and configuration may be modified. The software mainly includes two parts: logic region and physical region. Logic regional configuration needs topology network. Physical region mainly provides physical dimensions for network operation to guarantee rational analysis of basic composition and principles of computer network structure[2].

\section{Combination of computer network teaching and network simulation}

Organic combination of respective characteristics of network simulation technology and computer network teaching and rational introduction of computer networks technology in technology teaching can effectively help students well comprehend abstract theories which are difficult to understand in practical study. The development of network simulation technology can promote more vivid analysis of computer network. Computer Networks[Third Edition] gives an outline of ARP theory: how to map address to computer network data chain. For example, certain mapping with Ethernet can rationally optimize ARP and ensure improvement of work efficiency. If a computer has used ARP, certain results can be cached rationally to make sure they can continue to be used when the computer starts up next time. IP/TCP ARP can directly map original IP at the third layer to MAC at the second layer. The equipment which wants to jointly complete such step will respond to some extent. In this way, the desired MACT address can be cached in ARP. In a bid to get MAC address with better operation status, ARP will diffuse quickly in LAN. All pieces of equipment connecting LAN will independently cache certain ARP, or RAM is rationally utilized to store certain ARP results. Within certain time, ARP cache timer will no longer apply deleted ARP items. If students only listen to the teacher to explain ARP theory in classroom, the teacher needs to spend more time explaining, but also needs to spend more energy and time making students understand. If the teacher can rationally apply network simulation technology to establish certain model in computer network teaching, network content can be explained more vividly to gain good effects.

\section{Practical case of network simulation tool NS2}

\section{Basic overview of NS2}

After network simulation technology appeared, all kinds of network simulation tools may be gradually applied. At present, among existing network simulation tools, there are special tools to test network protocol algorithm and special tools to simulate specific environment network. Besides, there are also common network protocol simulation tools, such as OPNET and NS2. In current stage, OPNET and NS2 are commonly-used network simulation tools. OPNET is commercial software and owns high procurement cost. Generally, it is seldom applied in computer network teaching.

NS2 belongs to network simulation software of multi-protocol open source code. Through development and researches for many years, it is added in VINT project. NS2 continuously absorbs experience and achievements, mainly including CMU, UCB and other wireless network codes. Thus, it is necessary to continuously perfect and update NS2. In addition, NS2 also belongs to object-oriented simulated network environment tool driven by discrete event and ensures to simulate thorough network environment. NS2 mainly utilizes C++ class library and forms some common network link or networking protocol model, such as wireless network or wired network UDP, TCP, multicast and route protocol models. Practical cases which form according to the above class library can form corresponding network model and own certain details.

\section{Working process and principle of NS2}

NS2 uses two programming languages: $\mathrm{C}++$ and $\mathrm{OTcl}$ (own certain object-oriented Tcl design script program language). The above programming languages can offer two conditions for network simulation. Firstly, implement and simulate protocol specifically. High efficiency is required when designing header information and processing byte. In other words, running speed is mot important. 
Operating simulation environment searching, time and time of repairing loophole and re-compiling are not very important. On this basis, $\mathrm{C}++$ language is suitable. Secondly, during studying and analyzing network, the main basis is to alter environment and set up parameters and network modules. It is necessary to establish the required computer network environment quickly in a short time, discover ands modify. Thus, it is very important to set up network environment events. So, Tcl scripting language can completely accord with actual demand during deisgn[3].

TclCL is applied to rationally correlate variables and objects of OTcl and $\mathrm{C}++$ languages. The object and class of OTcl belong to explanatory object and explanatory class. The object and class of $\mathrm{C}++$ are compiling object and compiling class. TclCL is established and encapsulate on the basis of OTcl. For those using NS2, OTcl scripting language can reflect network topology. Based on Tcl foundation framework, OTc can extend object orientation and utilize $\mathrm{C}++$ to rationally compile network modules and time scheduler. The $\mathrm{C}++$ at the bottom layer can be used to express the core NS2 is C++. The bridge connecting $\mathrm{C}++$ and OTcl is actually TclCL. Then, user interface is rationally connected to form NS2. Basic working process of NS2 is the process of simulating actual problems to be solved through programming with rational application of OTcl script. OTcl language interpreter is used for execution. NS2 simulation library is used for simulation and compiling. Then, actual results are simulated. Relevant simulation contents are analyzed according to records to form data visibility graph or network topology. For newly researched and developed protocol or special conditions, C++ code may be properly modified and programmed to offer certain new class library for design. The basic working process is as follows:

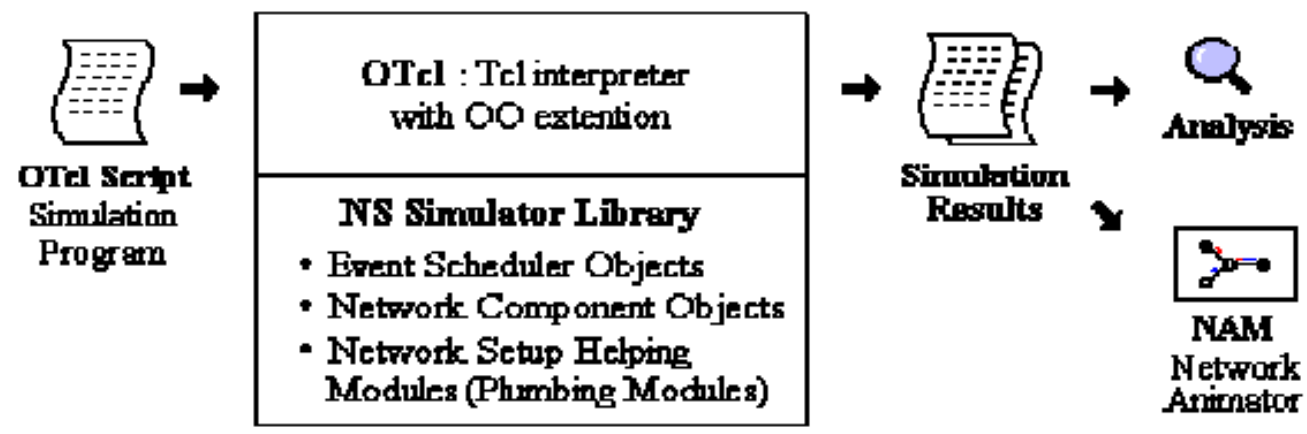

Fig. 1 Working process chart

\section{NS2 application in experiment teaching}

The main case is actually random and early detection of TCP congestion control. NS2 simulation tool is used for modeling to gain the corresponding operation results. The results are analyzed, and graph form is utilized to show data results to ensure more careful understanding of RED operation property and mechanism. RED belongs to early detection and stochastic algorithm of congestion avoidance mechanism. It will analyze each router during detecting queue. If congestion is found during detection, the source will be informed to properly adjust congestion window. The experimental condition is that certain topological structure is established, and RED queue is established between router $\mathrm{r} 1$ and router $\mathrm{r} 2$, including 25 data packages. It is required to analyze how RED utilizes measurement of actual dynamic length of average queue to avoid congestion after the experiment is completed. The data of simulation architecture are outputted according to graphical tool XGraph. Green line belongs to weighted dynamic average length, while red line belongs to instantaneous length. Analysis and detection are made to make sure students can more fully master how to operate RED, i.e. instantaneous length of RED queue which can fluctuate with the average length. Because some levels are lost in the queue, the above results appear. The results are grouped and discarded according to corresponding probability to avoid formation of congestion[4]. 


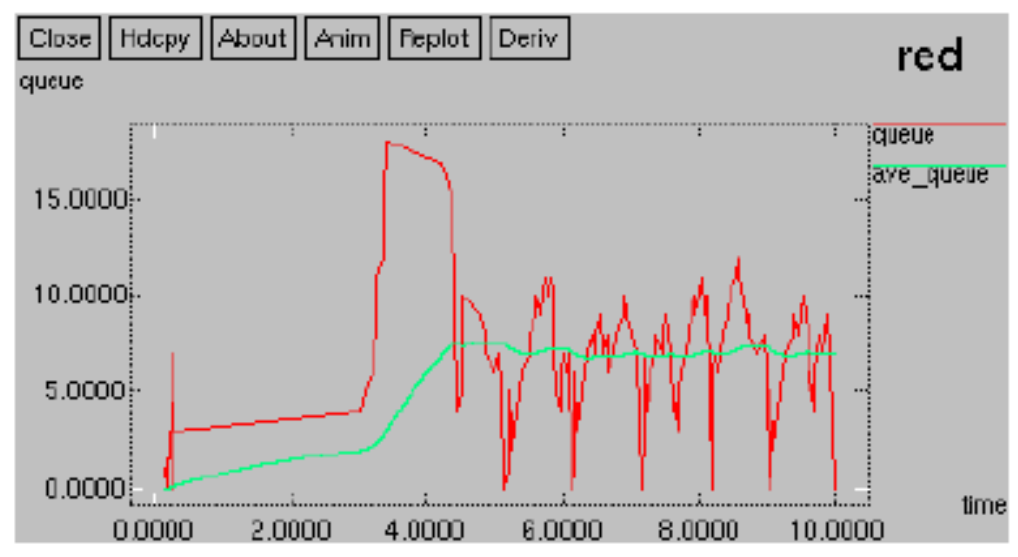

Fig. 2 Simulation data chart

\section{Verification through rational re-design or protocol alteration according to NS2}

In computer network teaching process, it is necessary to make sure students can fully comprehend fundamental and knowledge of operation of computer network, and guide students to rationally contrast existing network protocol according to experiments, fully master advantages and shortcomings of current protocol, encourage students to propose new protocol mode or properly improve existing protocol, boost students' enthusiasm and ensure certain creativity. How to establish new network protocol in NS2 and rationally verify new protocol is analyzed In accordance with simple example. The basic condition is as follows: if a multimedia procedure utilizes UDP for communication, and the people receiving and sending data need operate with different five transmission rates and codes in the way of automated negotiation. Meanwhile, actual situation of network congestion can be analyzed according to scale value. The five scale values are as follows: $0-4$. When sending starts, basic scale value is 0 . Adjustment of sending rate needs to be based on actual scale value returned. To better monitor network congestion, it is necessary to design detection and sending datagram with certain period. If detection data are lost within a period range, this indicates network congestion forms. It is necessary to properly reduce scale value of data transfer and decrease transmission rate and coding speed. If detection data will not be lost, proper improvement of scale value will improve transmission rate and coding speed. In the above experiment process, UDP mainly transmits datagram and distribute datagram. Current agency of datagram transfer only owns a head stack. Thus, current existing UPD agency should be specified. Datagram sending structure from the application layer should increase correspondingly. In the field where IP message header is not used, data type may be marked. According to actual analysis situation, $\mathrm{C}++$ programming language may be applied to properly alter and design experimental conditions of current UPD agency of datagram transmission, and properly neglect specific code and length problems in the process of sending network data class and network data. When the final topological graph is designed, it is required to properly test UPD multimedia transmission procedure in design process so as to ensure to own network calculator design model and improve computer network teaching efficiency[5].

\section{Conclusions}

In one word, analysis of the above process and utilization of simulation tool can not just more deeply analyze computer network operation principle in the experiment. After contrastive analysis of experiment, students' learning interest can be motivated; students' innovation ability and innovation consciousness are cultivated; social actual development demand can be fully met. Rational application of network simulation technology in computer network teaching can give full play to the function of simulation technology and lay a foundation for future development of computer network teaching. 


\section{References}

[1] Liu Guang, Application of network simulation tool in college computer network teaching. Modern Educational Technology, 2010,19(10):111-113,106.

[2] Chen Jian, Application of network simulation tool in computer network teaching, World of Net Friends Cloud Education, 2014(16):158-158.

[3] Deng Zhixia, On application of network simulation software in computer network teaching case study of NS2. Computer Knowledge and Technology, 2011,07(3):667-668.

[4] Liu Yanyan, Li Yulong, Application of NS-2 network simulator in computer network teaching. Journal of Hebei Software Institute, 2011,13(3):62-64.

[5] Yang Chunyong, Pan Wenjun, Zhu Cuitao et al., Study on computer network course teaching and aided teaching method. Journal of Higher Correspondence Education (Natural Sciences), 2011(6):12-14. 